\title{
A CCG APPROACH TO FREE WORD ORDER LANGUAGES
}

\author{
Beryl Hoffman * \\ Dept. of Computer and Information Sciences \\ University of Pennsylvania \\ Philadelphia, PA 19104 \\ (hoffman@linc.cis.upenn.edu)
}

\section{INTRODUCTION}

In this paper, I present work in progress on an extension of Combinatory Categorial Grammars, CCGs, (Steedman 1985) to handle languages with freer word order than English, specifically Turkish. The approach I develop takes advantage of CCGs' ability to combine the syntactic as well as the semantic representations of adjacent elements in a sentence in an incremental manner. The linguistic claim behind my approach is that free word order in Turkish is a direct result of its grammar and lexical categories; this approach is not compatible with a linguistic theory involving movement operations and traces.

A rich system of case markings identifies the predicate-argument structure of a Turkish sentence, while the word order serves a pragmatic function. The pragmatic functions of certain positions in the sentence roughly consist of a sentence-initial position for the topic, an immediately pre-verbal position for the focus, and post-verbal positions for backgrounded information (Erguvanli 1984). The most common word order in simple transitive sentences is SOV (SubjectObject-Verb). However, all of the permutations of the sentence seen below are grammatical in the proper discourse situations.

(1) a. Ayşe gazeteyi okuyor.

Ayşe newspaper-acc read-present.

Ayse is reading the newspaper.

b. Gazeteyi Ayşe okuyor.

c. Ayşe okuyor gazeteyi.

d. Gazeteyi okuyor Ayşe.

e. Okuyor gazeteyi Ayşe.

f. Okuyor Ayşe gazeteyi.

Elements with overt case marking generally can scramble freely, even out of embedded clauses. This suggest a CCG approach where case-marked elements are functions which can combine with one another and with verbs in any order.

${ }^{*}$ I thank Young-Suk Lee, Michael Niv, Jong Park, Mark Steedman, and Michael White for their valuable advice. This work was partially supported by ARO DAAL03-89C-0031, DARPA N00014-90-J-1863, NSF IRI 90-16592, Ben Franklin 91S.3078C-1.
Karttunen (1986) has proposed a Categorial Grammar formalism to handle free word order in Finnish, in which noun phrases are functors that apply to the verbal basic elements. Our approach treats case-marked noun phrases as functors as well; however, we allow verbs to maintain their status as functors in order to handle object-incorporation and the combining of nested verbs. In addition, CCGs, unlike Karttunen's grammar, allow the operations of composition and type raising which have been useful in handling a variety of linguistic phenomena including long distance dependencies and nonconstituent coordination (Steedman 1985) and will play an essential role in this analysis.

\section{AN OVERVIEW OF CCGS}

In CCGs, grammatical categories are of two types: curried functors and basic categories to which the functors can apply. A category such as $\mathrm{X} / \mathrm{Y}$ represents a function looking for an argument of category $\mathrm{Y}$ on its right and resulting in the category $\mathrm{X}$. A basic category such as $\mathrm{X}$ serves as a shorthand for a set of syntactic and semantic features.

A short set of combinatory rules serve to combine these categories while preserving a transparent relation between syntax and semantics. The application rules allow functors to combine with their arguments. Forward Application ( $>$ ):

$$
X / Y \quad Y \Rightarrow X
$$

Backward Application $(<)$ :

$$
Y X \backslash Y \Rightarrow X
$$

In addition, CCGs include composition rules to combine together two functors syntactically and semantically. If these two functors have the semantic interpretation $\mathrm{F}$ and $\mathrm{G}$, the result of their composition has the interpretation $\lambda x F(G x)$.

Forward Composition $(>B)$ :

$$
X / Y \quad Y / Z \Rightarrow X / Z
$$

Backward Composition $(<B)$ :

$$
Y \backslash Z \quad X \backslash Y \Rightarrow X \backslash Z
$$

Forward Crossing Composition ( $>B x$ ):

$$
\mathrm{X} / \mathrm{Y} \quad Y \backslash Z \Rightarrow \mathrm{N} \backslash Z
$$

Backward Crossing Composition $(<B x)$ :

$$
\forall / Z \quad X \backslash Y \Rightarrow X / Z
$$




\section{FREE WORD ORDER IN CCGs}

\section{Representing Verbs:}

In this analysis, we represent both verbs and casemarked noun phrases as functors. In Karttunen's analysis (1986), although a verb is a basic element rather than a functor, its arguments are specified as subcategorization features of its basic element category. We choose to directly represent a verb's subcategorization in its functor category. An advantage of this approach is that at the end of a parse, we do not need an extra process to check if all the arguments of a verb have been found; this falls out of the combination rules. Also, certain verbs need to act as active functors in order to combine with objects without case marking.

Following a suggestion of Mark Steedman, 1 define the verb to be an uncurried function which specifies a set of arguments that it can combine with in any order. For instance, a transitive verb looking for a nominative case noun phrase and an accusative case noun phrase has the category $S \mid\{N n, N a\}$. The slash $\mid$ in this function is undetermined in direction; direction is a feature which can be specified for each of the arguments, notated as an arrow above the argument, e.g. $S \mid\{\stackrel{\leftarrow}{n}\}$. Since Turkish is not strictly verb final, most verbs will not specify the direction features of their arguments.

The use of uncurried notation allows great freedom in word order among the arguments of a verb. However, we will want to use the curried notation for some functors to enforce a certain ordering among the functors' arguments. For example, object nouns or clauses without case-marking cannot scramble at all and must remain in the immediately pre-verbal position. Thus, verbs which can take a so called incorporated object will also have a curried functor category such as $S|\{N n, N d\}|\{\overleftarrow{n}\}$ forcing the verb to first apply to a noun without case-marking to its immediate left before combining with the rest of its arguments.

\section{Representing Nouns:}

The interaction between case-marking and the ability to scramble in Turkish supports the theory that casemarked nouns act as functors. Following Steedman (1985), order-preserving type-raising rules are used to convert nouns in the grammar into functors over the verbs. The following rules are obligatorily activated in the lexicon when case-marking morphemes attach to the noun stems.

\section{Type Raising Rules:}

$$
\begin{aligned}
& \mathrm{N}+\text { case } \Rightarrow(v \mid\{\ldots\}) \mid\{\overrightarrow{v \mid\{\text { Ncase }, \ldots\}}\} \\
& \mathrm{N}+\text { case } \Rightarrow(v \mid\{\ldots\}) \mid\{\overrightarrow{v \mid \overrightarrow{\text { Ncase }, \ldots}\}\}}
\end{aligned}
$$

The first rule indicates that a noun in the presence of a case morpheme becomes a functor looking for a verb on its right; this verb is also a functor looking for the original noun with the appropriate case on its left. After the noun functor combines with the appropriate verb, the result is a functor which is looking for the remaining arguments of the verb. $v$ is actually a variable for a verb phrase at any level, e.g. the verb of the matrix clause or the verb of an embedded clause. The notation ... is also a variable which can unify with one or more elements of a set.

The second type-raising rule indicates that a casemarked noun is looking for a verb on its left. Our CCG formalism can model a strictly verb-final language by restricting the noun phrases of that language to the first type-raising rule. Since most, but not all, case-marked nouns in Turkish can occur behind the verb, certain pragmatic and semantic properties of a Turkish noun determine whether it can type-raise using either rule or is restricted to only the first rule.

\section{The Extended Rules:}

We can extend the combinatory rules for uncurried functions as follows. The sets indicated by braces in these rules are order-free, i.e. $\mathrm{Y}$ in the following rules can be any element in the set. ${ }^{1}$

Forward Application' ( $>$ ):

$$
X|\{\vec{Y}, \ldots\} \quad Y \Rightarrow X|\{\ldots\}
$$

Backward Application' (<):

$$
Y \quad X|\{\stackrel{\leftarrow}{Y}, \ldots\} \Rightarrow X|\{\ldots\}
$$

Using these new rules, a verb can apply to its arguments in any order, or as in most cases, the casemarked noun phrases which are type-raised functors can apply to the appropriate verbs.

Certain coordination constructions (such as SO and SOV, SOV and SO) force us to allow two typeraised noun phrases which are looking for the same verb to combine together. Since both noun phrases are functors, the application rules above do not apply. The following composition rules are proposed to allow the combining of two functors.

Forward Composition' $(>B)$ :

$$
X|\{\vec{Y}, \ldots 1\} \quad Y|\{\ldots 2\} \Rightarrow X \mid\{\ldots 1, \ldots 2\}
$$

Backward Composition' $(<B)$ :

$$
Y|\{\ldots 1\} \quad X|\{\overleftarrow{Y}, \ldots 2\} \Rightarrow X \mid\{\ldots 1, \ldots 2\}
$$

The following example demonstrates these rules in analyzing sentence (1)b in the scrambled word order Object-Subject-Verb: ${ }^{2}$

\footnotetext{
${ }^{1}$ We assume that a category $X \mid\{\}$ where \{\} is the empty set rewrites by some clean-up rule to just $I$.

${ }^{2}$ The bindings of the first composition are $r_{2}=r_{1}$, $\{\ldots 2\}=\{\vec{N} a, \ldots 1\}$.
} 


\begin{tabular}{|c|c|}
\hline Gazeteyi & Ayşe \\
\hline$v_{1}|\{\ldots 1\}|\left\{\overline{v_{1} \mid\{\overline{\mathrm{N}}, \ldots 1}\right\}$ & $\left\{v_{2}|\{\ldots 2\}|\left\{\overrightarrow{v_{2} \mid\left\{\overline{\mathrm{Nn}_{1}, \ldots 2}\right.}\right\}\right\}$ \\
\hline$\left(v_{1} \mid\{\ldots 1\}\right) \mid$ & $\left\{v_{1} \mid\{\overline{\mathrm{Nn}}, \overline{\mathrm{Na}}, \ldots 1\}\right\}$ \\
\hline
\end{tabular}

\section{LONG DISTANCE SCRAMBLING}

In complex Turkish sentences with clausal arguments, elements of the embedded clauses can be scrambled to positions in the main clause, i.e. long distance scrambling. Long distance scrambling appears to be no different than local scrambling as a syntactic and pragmatic operation. Generally, long distance scrambling is used to move an element into the sentenceinitial topic position or to background it by moving it behind the matrix verb.

(2) a.

Fatma [Ayşe'nın gittiğini] biliyor.

Fatma [Ayşe-gen go-ger-3sg-acc] know-prog.

Fatma knows that Ayşe went away.

b. Ayşe'nın Fatma [gittigini] biliyor.

Ayşe-gen Fatma [go-ger-acc] know-prog.

c. Fatma [gittiğini] biliyor Ayşe'nın.

Fatma [go-ger-acc] know-prog Ayşe-gen.

The composition rules allow noun phrases to combine regardless of whether or not they are the arguments of the same verb. The same rules allow two verbs to combine together. In the following, the semantic interpretation of a category is expressed following the syntactic category.

$$
\frac{\begin{array}{ll}
\text { go-nominal-acc } & \text { knows. } \\
S_{N a}:\left(g o^{\prime} y\right) \mid\{N g: y\} & S:\left(k n o w^{\prime} p x\right) \mid\left\{N n: x, S_{N a}: p\right\}
\end{array}<\mathrm{B}}{S:\left(k_{n o w}\left(g o^{\prime} y\right) x\right) \mid\{N g: y, N n: x\}}
$$

As the two verbs combine, their arguments collapse into one argument set in the syntactic representation. However, the verbs' respective arguments are still distinct within the semantic representation of the sentence. The predicate-argument structure of the subordinate clause is embedded into the semantic representation of the matrix clause.

Long distance scrambling in Turkish is quite free; however, there are many pragmatic and processing constraints. A syntactic restriction may be needed to explain why elements in certain adjunct clauses (though not all) are very hard to long distance scramble. To account for these clauses, we can assign the head of the restricted adjunct clause a curried functor category such as $X|X|\{$ arguments... $\}$ rather than $X \mid\{X$, arguments... $\}$. The curried category forces the adjunct head to combine with all of its arguments in the adjunct clause before combining with the constituent it modifies. This blocks long distance scrambling out of that adjunct clause.
As mentioned before, another use for curried functions is with object nouns or clauses without case marking which are forced to remain in the immediately pre-verbal position. A matrix verb can have a category such as $S|\{N n\}|\left\{S_{2}\right\}$ to allow it to combine with a subordinate clause without case-marking $\left(S_{2}\right)$ to its immediate left. However, to restrict a type-raised $N n$ from interposing in between the matrix verb and the subordinate clause, we must restrict type raised noun phrases and verbs from composing together. A language specific restriction, allowing composition only if $(X \neq v \mid \ldots)$ or $(Y=v \mid \ldots)$, is proposed, similar to the one placed on the Dutch grammar by Steedman (1985), to handle this case.

\section{CONCLUSIONS}

What I have described above is work in progress in developing a CCG account of free word order languages. We introduced an uncurried functor notation which allowed a greater freedom in word order. Curried functors were used to handle certain restrictions in word order. A uniform analysis was given for the general linguistic facts involving both local and long distance scrambling. I have implemented a small grammar in Prolog to test out the ideas presented in this paper.

Further research is necessary in the handling of long distance scrambling. The restriction placed on the composition rules in the last section should be based on syntactic and semantic features. Also, we may want to represent subordinate clauses with casemarking as type-raised functions over the matrix verb in order to distinguish them from clauses without case-marking.

As a related area of research, prosody and pragmatic information must be incorporated into any account of free word order languages. Steedman (1990) has developed a categorial system which allows intonation to contribute information to the parsing process of CCGs. Further research is necessary to decide how best to use intonation and pragmatic information within a CCG model to interpret Turkish.

\section{References}

[1] Erguvanli, Eser Emine. 1984. The Function of Word Order in Turkish Grammar. University of California Press.

[2] Karttunen, Lauri. 1986. 'Radical Lexicalism'. Paper presented at the Conference on Alternative Conceptions of Phrase Structure, July 1986, New York.

[3] Steedman, Mark. 1985. 'Dependency and Coordination in the Grammar of Dutch and English', Language, 61, 523-568.

[4] Steedman, Mark. 1990. 'Structure and Intonation', MS-CIS-90-45, Computer and Information Science, University of Pennsylvania. 